\title{
Control of systems to sets and their interiors*
}

\author{
F. H. Clarke ${ }^{\dagger} \quad$ P. R. Wolenski ${ }^{\ddagger}$
}

CRM-2227

November 1994

*Work supported by the Natural Sciences and Engineering Research Council of Canada and by the fonds FCAR du Québec.

†Centre de recherches mathématiques, Université de Montréal, C.P. 6128, Succ Centre-ville Montréal, Québec, Canada, H3C 3J7

${ }^{\ddagger}$ Department of Mathematics, Louisiana State University, Baton Rouge, Louisiana 70803, U.S.A. 


\begin{abstract}
For a closed target set $S \subset \mathbb{R}^{n}$ and a control system (formulated as a differential inclusion and defined near $S$ ), the present paper considers a sufficient condition for the property that every point near $S$ can be steered to $S$ in finite time by some trajectory of the system. Estimates are obtained revealing how fast some such trajectory is nearing the target. A strong form of this condition is shown to imply that every trajectory of the system hits the target. With a further assumption on the target set $S$, we also consider conditions that guarantee that some trajectories enter the interior of $S$.
\end{abstract}

Keywords. Weak and strong attainability, control systems, penetrative systems, minimum time function

\title{
Résumé
}

On présente des conditions assurant qu'un système contrôle puisse être dirigé vers un ensemble en temps fini. La question de pénétrer l'intérieur d'un ensemble est également étudiée. L'outil principal est l'analyse non lisse, en particulier les méthodes proximales. 


\section{Introduction}

This paper presents conditions on a control system and a target set $S$ that guarantee that any point near $S$ can be steered to $S$ in finite time by at least one trajectory of the system. We call such a system and target set weakly attainable. Similar considerations are made for strongly attainable systems, in which all trajectories hit the target. Furthermore, in a similar vein, we consider conditions which allow for arcs to penetrate into the interior of $S$.

Our results are local in nature, and thus the target set $S \subset \mathbb{R}^{n}$ is assumed to be compact. We shall formulate the control system as a differential inclusion

$$
\begin{aligned}
& \dot{x}(t) \in F(x(t)) \text { a.e. } t \in[0, T] \\
& x(0)=x,
\end{aligned}
$$

in which $F: \mathbb{R}^{n} \rightrightarrows \mathbb{R}^{n}$ is a multifunction with compact convex values. A sufficient condition for weak attainability (see Theorem 3.1 below) is that $F$ be continuous and that there exists $\delta>0$ so that for every $y \in \operatorname{bd} S$ and $\zeta \in N_{S}^{P}(y)$, we have

$$
\min _{v \in F(y)}\langle\zeta, v\rangle \leq-\delta\|\zeta\|
$$

For strong attainability, the "min" in (1) is replaced by "max". The notation $N_{S}^{P}(y)$ refers to the proximal normal cone at $y$ (see Section 2). Notice that condition (1) (the type of which we refer to as a $\delta$ condition) is imposed only at points on the boundary of $S$, and is nonvacuous at only those points that have a nonzero proximal normal. Condition (1) reduces to the one introduced by Petrov (Ref. 1) (with $S=$ the origin), but which is stated to hold in a neighborhood of the target. More recently, Bardi and Falcone (Ref. 2) and Cannarsa and Sinestrari (Ref. 3) have considered such local assumptions with general closed target sets, but the dynamics require at least Lipschitz state dependence. We show in Theorem 3.4 that our continuity assumption on $F$ can be further weakened to upper semicontinuity if the $\delta$ condition (1) is assumed to hold for all $y$ near $S$ and for any $\zeta \in y-\operatorname{proj}_{S}(y)$, where $\operatorname{proj}_{S}(y)$ are those elements in $S$ closest to $y$. Both continuity and upper semicontinuity are new in this context

The proofs of our attainability results are based upon a time discretization algorithm, which can accurately be described as proximal projection. This in turn consists of applying the mean value inequality (Ref. 4) in conjunction with new results in (Ref. 5) on properties of the distance function to a closed set. Our essentially algorithmic approach appears to yield the best rate of convergence among the existing ones.

Dynamic programming techniques are used in (Ref. 2) to perform an approximation to the minimum time function, which is defined at a point $x$ as the least time $T$ for which a solution $x(\cdot)$ to (1) exists and has $x(T) \in S$. More specifically, a discretization of the associated Hamilton-Jacobi equation is made in (Ref. 2), and then it is shown that the discrete solutions converge to the so-called viscosity solution. The discretization is in fact an approximation to the minimum time function because the latter is the viscosity solution (which is unique) of the Hamilton-Jacobi equation. A major difference with proximal projection as developed here from a method based on dynamic programming is that the former is moving "forward" in time, while the latter uses a "backward" construction. The appendix in (Ref. 3) also uses a forward time procedure, somewhat similar in nature to ours, but is only applicable for problems formulated with explicit control variables without feedback. For the goals of that paper, further smoothness in the state variable is also required.

After giving some preliminaries in Section 2, we prove the attainability results in Section 3. Under an additional epi-continuous assumption on the target set $S$, we show in Section 4 that there exist trajectories entering the interior of $S$. Our results appear to be the first on this issue. Examples are provided in Section 5 illustrating the role of the hypotheses. 


\section{Preliminaries}

Let $S \subset \mathbb{R}^{n}$ be compact, and $F: \mathbb{R}^{n} \rightrightarrows \mathbb{R}^{n}$ be a multifunction with compact convex values. Recall that $F$ is said to be continuous of modulus $\omega_{F}$ near $S$ provided

$$
\Delta_{H}(F(x), F(y)) \leq \omega_{F}(\|x-y\|) \text { for all } x, y \text { near } S
$$

where $\Delta_{H}$ denotes the Hausdorff metric, and $\omega_{F}:[0, \infty) \longrightarrow[0, \infty)$ is a continuous, nondecreasing function with $\omega_{F}(0)=0$. $F$ is upper semicontinuous at $x$ if all sequences $\left\{y_{i}\right\},\left\{v_{i}\right\}$ satisfying $y_{i} \rightarrow x$ and $v_{i} \in F\left(y_{i}\right)$ have the property that every cluster point of $\left\{v_{i}\right\}$ lies in $F(x)$.

We next give the relevant background of proximal analysis featured in this paper. For a more complete treatment with applications to optimization, see Clarke (Ref. 6) and Loewen (Ref. 7). If $y \in \operatorname{bd} S$, a vector $\zeta \in \mathbb{R}^{n}$ is called a proximal normal to $S$ at $y$ provided there exists $r>0$ so that

$$
\{y+r(\zeta+\operatorname{cl} B)\} \cap S=\{y\} .
$$

The set of all proximal normals at a point $y$ is a cone, and is denoted by $N_{S}^{P}(y)$.

Now suppose $f: \mathbb{R}^{n} \longrightarrow \mathbb{R}^{1} \cup\{+\infty\}$ is lower semicontinuous, and $x \in \mathbb{R}^{n}$ is such that $f(x)<+\infty$. A vector $\zeta \in \mathbb{R}^{n}$ is called a proximal subgradient of $f$ at $x$ provided that $(\zeta,-1) \in$ $N_{\text {epif }}^{P}(x, f(x))$, where

$$
\text { epi } f:=\{(y, \alpha): \alpha \geq f(y)\}
$$

is the epigraph of $f$. The set of proximal subgradients of $f$ at $x$ is denoted by $\partial_{P} f(x)$. One can show that $\zeta \in \partial_{P} f(x)$ if and only if there exists $\sigma>0$ so that

$$
f(y)-\langle\zeta, y-x\rangle+\sigma\|y-x\|^{2} \geq f(x)
$$

for all $y$ near $x$.

We shall require the following theorem from the calculus governing proximal subgradients, which is labeled as the mean value inequality (Clarke and Ledyaev (Ref. 4), (Ref. 8)). Suppose $Y$ is compact and convex, and $x_{0}$ is such that $f\left(x_{0}\right)<\infty$. We write $\left[x_{0}, Y\right]$ for the convex hull of $x_{0}$ and $Y$.

Theorem 2.1 (Mean value inequality). Suppose $f$ is lower semicontinuous and bounded below on $\left[x_{0}, Y\right]+k B$ for some $k>0$. Then for any $\varepsilon>0$ there exists $z \in\left[x_{0}, Y\right]+\varepsilon B$ and $\zeta \in \partial_{P} f(z)$ such that

$$
\min _{Y} f-f(x)<\left\langle\zeta, y-x_{0}\right\rangle+\varepsilon \text { for all } y \in Y .
$$

Furthermore, $z$ can be chosen to satisfy

$$
f(z)<\inf _{\left[x_{0}, Y\right]} f+\left|\min _{Y} f-f(x)\right|+\varepsilon .
$$

We shall measure the rate at which a trajectory approaches $S$ by the so-called distance function $d_{S}$ to $S$, defined by

$$
d_{S}: \mathbb{R}^{n} \longrightarrow \mathbb{R}^{1} ; \quad d_{S}(x):=\inf _{y \in S}\|x-y\| .
$$

The set of elements in $S$ closest to $x \notin S$ is denoted by $\operatorname{proj}_{S}(x)$. That is,

$$
\operatorname{proj}_{S}(x)=\left\{y \in S: d_{S}(x)=\|x-y\|\right\} .
$$

We have recently carried out a detailed study of the differentiability properties of $d_{S}$ in (Ref. 5) and (Ref. 9). For the immediate purposes ahead, however, we shall only require the following formula for the proximal subgradient at points off $\mathrm{S}$, which is proven in (Ref. 5). 
Theorem 2.2. Suppose $x \notin S$ and $\zeta \in \partial_{P} d_{S}(x)$. Then $\operatorname{proj}_{S}(x)$ consists of a single element $y$, say, and

$$
\zeta=(x-y) / d_{S}(x)
$$

The main concepts in this paper are precisely defined in the following definitions. We suppose that $S$ is compact, and that $F$ has compact convex values and is at least upper semicontinuous.

Definition 2.1. (a) The pair $(S, F)$ is called weakly attainable of radius $r>0$ provided that for every $x \in S+r B$, there exist a time $T>0$ and a trajectory $x(\cdot)$ of $F$ defined on $[0, T]$ for which $x(0)=x$ and $x(T) \in S$. If in addition there exists a constant $c>0$ for which

$$
d_{S}(x(t)) \leq d_{S}(x(s))-(t-s) c \text { for all } 0 \leq s<t \leq T
$$

then $c$ is called a rate of weak attainability of $(S, F)$.

(b) The pair $(S, F)$ is called strongly attainable of the corresponding radius and rate if the properties in (a) hold for every trajectory of $F$ (but of course for perhaps different $T$ ).

We denote the closure of the complement of $S$ by $\overline{\operatorname{comp}} S$. It is convenient for our purposes in Section 4 to state the penetration definitions as originating from a fixed point on the boundary, rather than making a simultaneous declaration at all points of the boundary.

Definition 2.2. (a) The pair $(S, F)$ is weakly penetrative at $x \in \operatorname{bd} S$ provided there exists a trajectory $x(\cdot)$ of $F$ for which $x(0)=x$ and $d_{\overline{\operatorname{comp}} S}(x(t))>0$ for all small $t>0$. In this case, a value $r>0$ so that the trajectory can be chosen on an interval $[0, T]$ with $d_{\overline{\operatorname{comp}} S}(x(T)) \geq r$ is a radius of penetration. If a constant $c$ is also available satisfying

$$
x(t)+t c B \subset S \text { for all } 0 \leq t \leq T,
$$

then $c$ is called a rate of penetration at $x$.

(b) The pair $(S, F)$ is called strongly penetrative at $x \in \mathrm{bd} S$ of the corresponding size and rate if the properties in (a) hold for every trajectory of $F$ originating from $x$.

\section{Attainable systems}

The main result of this section is the following.

Theorem 3.1. Suppose $S \subseteq \mathbb{R}^{n}$ is nonempty and compact, and $F: \mathbb{R}^{n} \rightrightarrows \mathbb{R}^{n}$ is a continuous multifunction of modulus $\omega_{F}$ with compact convex values. Suppose there exists $\delta>0$ so that whenever $y \in S$ and $\zeta \in N_{S}^{P}(y)$, there exists $v \in F(y)$ for which

$$
\langle\zeta, v\rangle \leq-\delta\|\zeta\|
$$

Then $(S, F)$ is weakly attainable. Moreover, the radius and rate of attainability depend only on $\delta$ and $\omega_{F}$.

Proof. We first fix the constants featured in the estimates to come. Let

$$
\begin{aligned}
r, c & >0 \quad \text { so that } \quad \delta / 2-\omega_{F}(2 r) \geq c>0 \\
T & :=r / c \\
M & :=\sup \{\|v\|: v \in F(x), x \in S+r \operatorname{cl} B\} .
\end{aligned}
$$


Let $x \in S+r B$. Now let $N$ be a sufficiently large positive integer so that $h:=T / N$ satisfies

$$
h<\min \left\{r /(2(M+\delta)),\left(r-d_{S}(x)\right) / M\right\} .
$$

We shall proceed by constructing a sequence of length no more than $N$ for which the last element will lie within a distance of $(h \delta) / 2$ of $S$.

The Mean Value Inequality applied with data $f:=d_{S}, Y:=x_{0}+h F\left(x_{0}\right)$ and $\varepsilon:=(h \delta) / 2$ provides a point $z$ and a vector $\zeta$ in such a manner that

$$
\begin{aligned}
\zeta & \in \partial_{P} d_{S}(z) \\
z & \in\left[x_{0}, x_{0}+h F\left(x_{0}\right)\right]+\varepsilon B, \\
d_{S}(z) & \leq d_{S}\left(x_{0}\right)+h M+\varepsilon
\end{aligned}
$$

and that the following inequality holds for all $w \in F\left(x_{0}\right)$ :

$$
\min _{v \in F\left(x_{0}\right)} d_{S}\left(x_{0}+h v\right)-d_{S}\left(x_{0}\right)<\langle\zeta, h w\rangle+\varepsilon .
$$

Our construction will terminate when $z$ lands in $S$, so let us suppose for the moment that $z \notin S$. By Theorem 2.2, we have from (2) that

$$
\zeta=(z-y) / d_{S}(z)
$$

where $\{y\}=\operatorname{proj}_{S}(z)$, which immediately implies that

$$
\zeta \in N_{S}^{P}(y) \text { and }\|\zeta\|=1
$$

From the $\delta$ assumption in the theorem applied to $\zeta$ as in (6), there exists $v \in F(y)$ so that

$$
\langle\zeta, v\rangle \leq-\delta
$$

The continuity hypothesis on $F$ implies the existence of some $w \in F\left(x_{0}\right)$ so that

$$
\|w-v\| \leq \omega_{F}\left(\left\|y-x_{0}\right\|\right)
$$

Hence from (5), we see that

$$
\begin{aligned}
\min _{v^{\prime} \in F\left(x_{0}\right)} d_{S}\left(x_{0}+h v^{\prime}\right)-d_{S}\left(x_{0}\right) & <h\langle\zeta, w\rangle+\varepsilon \\
& \leq h\langle\zeta, v\rangle+h\langle\zeta, w-v\rangle+\varepsilon \\
& \leq-\delta h+h \omega_{F}\left(\left\|y-x_{0}\right\|\right)+\varepsilon
\end{aligned}
$$

where we have used (6), (7), and (8) in deducing the last inequality. We see from (3) and (4) that

$$
\begin{aligned}
\left\|y-x_{0}\right\| & \leq\|y-z\|+\left\|z-x_{0}\right\| \\
& \leq d_{S}(z)+h M+\varepsilon \\
& \leq d_{S}\left(x_{0}\right)+2(h M+\varepsilon) \\
& \leq r+2 h(M+\delta) \\
& \leq 2 r .
\end{aligned}
$$


The last inequality follows from the choice of $N$. Since modulus functions are nondecreasing, we immediately have from (10) that

$$
\omega_{F}\left(\left\|y-x_{0}\right\|\right) \leq \omega_{F}(2 r)
$$

Inserting (11) into (9), we deduce from (9) and our choices of $\varepsilon$ and $c$ that there exists $v_{0} \in F\left(x_{0}\right)$ so that

$$
\begin{aligned}
d_{S}\left(x_{0}+h v_{0}\right)-d_{S}\left(x_{0}\right) & <\varepsilon-\delta h+h \omega_{F}(2 r) \\
& \leq h\left(\omega_{F}(2 r)-\delta / 2\right) \\
& \leq-h c .
\end{aligned}
$$

Now let $x_{1}=x_{0}+h v_{0}$, and apply the above procedure initialized at the point $x_{1}$ in place of $x_{0}$. Continuing in this manner, a sequence $x_{0}, x_{1}, x_{2}, \ldots$ is obtained for which

$$
d_{S}\left(x_{i+1}\right)-d_{S}\left(x_{i}\right) \leq-h c
$$

holds for each $i$ (as (12) holds in the case $i=0$ ), and for which the choice of $z$ does not lie in $S$. When $z \in S$, the process is terminated. Let us next observe that the termination must occur at some step $i_{N}$ that cannot be larger than $N$. Indeed, at each step before termination, (13) holds, and with $i$ applications yields that

$$
\begin{aligned}
0 \leq d_{S}\left(x_{i}\right) & \leq d_{S}\left(x_{i-1}\right)-c h \\
& \leq d_{S}\left(x_{0}\right)-(i c T) / N \\
& \leq(1-i / N) r .
\end{aligned}
$$

Hence we must have $i_{N} \leq N$.

In the terminating step $i_{N}$ in which $z \in S$, we have

$$
z \in\left\{\left[x_{i_{N}-1}, x_{i_{N}-1}+h F\left(x_{i_{N}-1}\right)\right]+\varepsilon\right\} \cap S
$$

and choose

$$
v_{i_{N}-1} \in F\left(x_{i_{N}-1}\right) \quad \text { and } \quad h^{\prime} \in[0, h]
$$

so that $x_{i_{N}}:=x_{i_{N}-1}+h^{\prime} v_{i_{N}-1}$ satisfies $\left\|z-x_{i_{N}}\right\|<\varepsilon$. Consequently the last element $x_{i_{N}}$ in the sequence satisfies

$$
d_{S}\left(x_{i_{N}}\right)<\varepsilon \leq(h \delta) / 2 .
$$

The entire sequence $x_{1}, x_{2}, \ldots, x_{i_{N}}$ of course depends on $N$. Let $h_{N}^{\prime}$ be the $h^{\prime}$ value found in (15), and let $T_{N}:=T i_{N}-1 / N+h_{N}^{\prime}$. Define an $\operatorname{arc} x^{N}(\cdot)$ on $\left[0, T_{n}\right]$ as the linear interpolation of these points, which means precisely that

$$
x^{N}(t):=\left\{\begin{array}{r}
x_{i}+(t-(i T) / N)\left(x_{i+1}-x_{i}\right)(1 / h) \\
\text { if } \quad t \in[(i-1) T / N,(i) T / N] \\
\text { and } 1 \leq i<i_{N} \\
x_{i_{N}-1}+\left(t-\left(i_{N}-1\right) T / N\right)\left(x_{i_{N}}-x_{i_{N}-1}\right)(1 / h) \\
\text { if } t \in\left[\left(i_{N}-1\right) T / N, T_{N}\right]
\end{array}\right.
$$


We pass to a subsequence $\left\{N_{j}\right\}$ so that $T_{N_{j}} \rightarrow T^{\prime}$ as $j \rightarrow \infty$ for some $T^{\prime} \in(0, T]$, and so that $x^{N_{j}}(t) \rightarrow x(t)$ uniformly over $t \in\left[0, \min \left\{T_{N_{j}}, T^{\prime}\right\}\right]$, where $x(\cdot)$ is a trajectory for $F$ on $\left[0, T^{\prime}\right]$. The convergence of the arcs is assured by the compactness of trajectories theorem (see (Ref. 10), Theorem 3.1.7). From (16), we see that

$$
d_{S}\left(x\left(T^{\prime}\right)\right)=\lim _{j \rightarrow \infty} d_{S}\left(x^{N_{j}}\left(T_{N_{j}}\right)\right)=0
$$

which says that $x\left(T^{\prime}\right) \in S$.

The radius of attainability has thus been shown to be $r$, which depends only on $\delta$ and $\omega_{F}$. To verify the rate assertion in the theorem, let $0 \leq s<t \leq T^{\prime}$. Let $s_{j}$ and $t_{j}$ be grid elements of the form $k T / N_{j}$, and so that $s_{j} \rightarrow s$ and $t_{j} \rightarrow t$ as $j \rightarrow \infty$. We have by (13) that

$$
d_{S}\left(x_{N_{j}}\left(t_{j}\right)\right)-d_{S}\left(x_{N_{j}}\left(s_{j}\right)\right) \leq-\left(t_{j}-s_{j}\right) c .
$$

Letting $j \rightarrow \infty$ in (17) yields that

$$
d_{S}(x(t)) \leq d_{S}(x(s))-(t-s) c
$$

which is the rate assertion in the theorem.

Remark 3.1. Suppose the pair $(S, F)$ is given and $x \notin S$. The minimum time $T_{S}(x)$ that it takes to reach $S$ from $x$ is given by

$$
\begin{gathered}
T_{S}(x):=\min \{T: \text { there exists a trajectory } x(\cdot) \text { for } F \text { on }[0, T] \\
\text { so that } x(0)=x \text { and } x(T) \in S\}
\end{gathered}
$$

It is immediate from the definitions that if $(S, F)$ has a radius of attainability of $r$ and a rate of attainability of $c$, then

$$
T_{S}(x) \leq d_{S}(x) / c
$$

for all $x \in S+r B$. We record this fact in the next corollary as it pertains to the data in Theorem 3.1.

Corollary 3.1. Suppose the hypotheses of Theorem 3.1 hold, and $x \in S+r B$. Then (18) holds.

We now turn to sufficient conditions for strong attainability.

Theorem 3.2. Suppose $S \subseteq \mathbb{R}^{n}$ is compact and $F: \mathbb{R}^{n} \rightrightarrows \mathbb{R}^{n}$ is a continuous multifunction of modulus $\omega_{F}$ with compact convex values. Suppose there exists $\delta>0$ so that for every $y \in S$, $\zeta \in N_{S}^{P}(y)$, and $v \in F(y)$ we have

$$
\langle\zeta, v\rangle \leq-\delta\|\zeta\|
$$

Then $(S, F)$ is strongly attainable. Moreover, the radius and rate of attainability depend only on $\delta$ and $\omega_{F}$.

Proof. We shall show that for $t \in\left[0, \frac{r}{c}\right]$, the inequality

$$
d_{S}(x(t))-d_{S}(x) \leq-c t
$$


holds for every trajectory $x(\cdot)$ of $F$ on $[0, r / c]$ that begins at $x \in S+r B$, where the constants $c$ and $r$ are chosen as in the proof of Theorem 3.1. This suffices to prove the theorem.

Let $t<r / c, x \in S+r B$, and $x(\cdot)$ a trajectory for $F$ that begins at $x$. We apply a simple instance of the Mean Value Inequality, the data taken as $f:=d_{S}, x_{0}:=x, Y:=\{x(t)\}$, and $\varepsilon:=t \delta / 2$. We so obtain $z$ and $\zeta$ satisfying

$$
\begin{aligned}
\zeta & \in \partial_{P} d_{S}(z) \\
z & \in[x, x(t)]+\varepsilon B \\
d_{S}(z) & \leq d_{S}\left(x_{0}\right)+h M+\varepsilon
\end{aligned}
$$

and such that

$$
d_{S}(x(t))-d_{S}(x)<\langle\zeta, x(t)-x\rangle+\varepsilon
$$

Let $y$ be such that $\{y\}=\operatorname{proj}_{S}(z)$, and let $v(\cdot)$ be chosen so that for almost all $s \in[0, t]$, we have $v(s) \in F(y)$ and

$$
d_{F(y)}(\dot{x}(s))=\|v(s)-\dot{x}(s)\| .
$$

Then $v(\cdot)$ is measurable and bounded, and the convexity of $F(y)$ assures that

$$
v:=(1 / t) \int_{0}^{t} v(s) d s
$$

belongs to $F(y)$. Hence by assumption, we have

$$
\langle v, \zeta\rangle \leq-\delta
$$

We also have that

$$
\begin{aligned}
\left\|v-\frac{x(t)-x}{t}\right\| & \leq \frac{1}{t} \int_{0}^{t}\|v(s)-\dot{x}(s)\| d s \\
& \leq \frac{1}{t} \int_{0}^{t} \omega_{F}(\|y-x(s)\|) d s \\
& \leq \omega_{F}(2 r),
\end{aligned}
$$

where the last inequality is obtained as in (10). We now have that

$$
\begin{aligned}
d_{S}(x(t))-d_{S}(x) & \leq\langle\zeta, x(t)-x\rangle+\varepsilon & & (\text { by }(20)) \\
& \leq t\langle\zeta, v\rangle+t \omega_{F}(2 r)+t \frac{\delta}{2} & & (\text { by }(22),\|\zeta\|=1) \\
& \leq t\left(\omega_{F}(2 r)-\frac{\delta}{2}\right) & & (\text { by }(21)) \\
& \leq-c t, & &
\end{aligned}
$$

which finishes the proof of the theorem.

The above sufficient conditions for attainability have contained a constant $\delta$ that must be independent of the particular $y \in \mathrm{bd} S$. As we see below in Example 5.2, such uniformity in the choice of $\delta$ is required. However, in the next result, we give a condition which must only be verified pointwise, although it is now nonvacuous on each boundary point of $S$. To state the result, we need a few more concepts from nonsmooth analysis. 
Let $y \in S$. The limiting normal cone $N_{S}^{L}(y)$ of $S$ at $y$ is defined as

$$
\begin{gathered}
N_{S}^{L}(y):=\left\{\zeta: \text { there exist sequences }\left\{y_{i}\right\},\left\{\zeta_{i}\right\} \text { so that } y_{i} \rightarrow y, \zeta_{i} \rightarrow \zeta,\right. \\
\text { and } \left.\zeta_{i} \in N_{S}^{P}\left(y_{i}\right) \text { for each } i\right\} .
\end{gathered}
$$

The (Clarke, complete, or convexified) normal cone $N_{L}^{C}(y)$ is defined as the closed convex hull of $N_{S}^{L}(y)$. Finally, the tangent cone $T_{S}^{C}(y)$ of $S$ at $y \in S$ is the polar of $N_{L}^{C}(y)$. That is,

$$
T_{S}^{C}(y):=\left\{v:\langle\zeta, v\rangle \leq 0 \text { for every } \zeta \in N_{L}^{C}(y)\right\}
$$

See (Ref. 10) for additional properties and characterizations of $T_{S}^{C}$.

The next result gives sufficient conditions for attainability in terms of the tangent cone. The interior of the tangent cone is denoted by int $T_{S}^{C}(y)$.

Theorem 3.3. Suppose for each $y \in S$, we have

$$
F(y) \cap \operatorname{int} T_{S}^{C}(y) \neq \phi .
$$

Then $(S, F)$ is weakly attainable. If we have

$$
F(y) \subseteq \operatorname{int} T_{S}^{C}(y)
$$

for each $y \in S$, then $(S, F)$ is strongly attainable.

Proof. Fix $y \in \operatorname{bd} S$. By assumption, we can choose $v_{y} \in F(y)$ and $\delta_{y}>0$ so that

$$
v_{y}+\delta_{y} B \subset T_{S}^{C}(y)
$$

Thus for any $\zeta \in N_{S}^{C}(y)$, we have that

$$
\begin{aligned}
\min _{v \in F(y)}\langle\zeta, v\rangle & \leq\left\langle\zeta, v_{y}\right\rangle \\
& \leq \min _{b \in B}\left\{-\left\langle\zeta, \delta_{y} b\right\rangle+\left\langle\zeta, v_{y}+\delta_{y} b\right\rangle\right\} \\
& \leq-\delta_{y}\|\zeta\| .
\end{aligned}
$$

Let $\rho_{y}>0$ be chosen so that $\omega_{F}\left(\rho_{y}\right)<\delta_{y} / 2$. Notice that $(23)$ and the continuity assumption on $F$ implies that for any $z \in y+\rho_{y} B$, we have

$$
\begin{aligned}
\min _{w \in F(z)}\langle\zeta, w\rangle & \leq \min _{\substack{w \in F(z) \\
v \in F(y)}}\{\langle\zeta, w-v\rangle+\langle\zeta, v\rangle\} \\
& \leq\left(\omega_{F}(\|z-y\|)-\delta_{y}\right)\|\zeta\| \\
& \leq-\left(\delta_{y} / 2\right)\|\zeta\|
\end{aligned}
$$

Now suppose $\left\{z_{i}\right\}$ is any sequence with $z_{i} \rightarrow y$ and for which there exists $0 \neq \zeta_{i} \in N_{S}^{P}\left(z_{i}\right)$. Passing to a subsequence if necessary, we assume there exists $\zeta$ so that $\zeta_{i} /\left\|\zeta_{i}\right\| \rightarrow \zeta \in N_{S}^{C}(y)$. By (23) and (24), we have

$$
\min _{w \in F\left(z_{i}\right)}\left\langle\zeta_{i}, w\right\rangle \leq-\left(\delta_{y} / 4\right)\left\|\zeta_{i}\right\|
$$


for all $i$ sufficiently large. Now (25) holds for arbitrary sequences $\left\{z_{i}\right\}$ and $\left\{\zeta_{i}\right\}$, and thus by shrinking $\rho_{y}$ if necessary, we may conclude that

$$
\min _{w \in F(z)}\langle\zeta, w\rangle \leq-\left(\delta_{y} / 4\right)\|\zeta\|
$$

for all $z \in y+\rho_{y} B$ and $\zeta \in N_{S}^{P}(z)$. We next observe that $\left\{y+\rho_{y} B\right\}_{y \in \operatorname{bd} S}$ is an open cover of bd $S$. A standard compactness argument yields that the hypotheses of Theorem 3.1 hold, the conclusion of which says that $(S, F)$ is weakly attainable.

The proof of the strong attainable assertion is similar.

The next attainability result states that the continuity assumption on $F$ can be weakened to upper semicontinuity provided a $\delta$ condition holds in a fixed neighborhood of $S$.

Theorem 3.4. Suppose $S \subseteq \mathbb{R}^{n}$ is compact and $F: \mathbb{R}^{n} \rightrightarrows \mathbb{R}^{n}$ is a multifunction with compact convex values and is upper semicontinuous on $S+r B$, where $r>0$. Suppose there exists $\delta>0$ so that whenever $y \notin S$ with $d_{S}(y)<r$, and $\zeta \in y-\operatorname{proj}_{S}(y)$, there exists $v \in F(y)$ for which

$$
\langle\zeta, v\rangle \leq-\delta\|\zeta\|
$$

Then $(S, F)$ is weakly attainable of radius $r$ and with rate $\delta$. If this inequality holds for all $v \in F(y)$, then $(S, F)$ is strongly attainable of radius $r$ and rate $\delta$.

Proof. Let us first assume that $F$ is Lipschitz continuous of rank $\lambda_{F}$, and we refer back to the proof of Theorem 3.1. The proximal projection algorithm can in fact be made simpler here because the $\delta$ assumption can be applied directly to the point $z$ obtained from the mean value inequality. One must however initially take potentially smaller values of $h$ and $\varepsilon$. We introduce another small parameter $\eta$ with $0<\eta<\delta$, and choose $h>0$ and $\varepsilon>0$ small enough to satisfy

$$
2 \lambda_{F}(h M+\varepsilon)<\eta / 2, \quad \varepsilon<(h \eta) / 2, \quad \text { and } \quad\left[x_{0}, x_{0}+h F\left(x_{0}\right)\right]+\varepsilon B \subset S+r B .
$$

Suppose $z$ and $\zeta$ are obtained to satisfy (2)-(5). Under the present hypothesis, which is active in the neighborhood $S+r B$ containing $z$, we are assured the existence of $v \in F(z)$ so that

$$
\langle\zeta, v\rangle \leq-\delta
$$

Let $w_{0} \in F\left(x_{0}\right)$ be so that $\left\|w_{0}-v\right\| \leq \lambda_{F}\left\|x_{0}-z\right\| \leq \lambda_{F}(h M+\varepsilon)$. Now by (5), (27), and the choices of $h$ and $\varepsilon$, we have

$$
\begin{aligned}
\min _{v^{\prime} \in F\left(x_{0}\right)} d_{S}\left(x_{0}+h v^{\prime}\right)-d_{S}\left(x_{0}\right) & \leq h\left\langle\zeta, w_{0}\right\rangle+\varepsilon \\
& =h\left\langle\zeta, w_{0}-v\right\rangle+h\langle\zeta, v\rangle+\varepsilon \\
& \leq-h \delta+h \lambda_{F}(h M+\varepsilon)+\varepsilon \\
& \leq-h(\delta-\eta) .
\end{aligned}
$$

Hence there exists $v_{0} \in F\left(x_{0}\right)$ so that

$$
d_{S}\left(x_{0}+h v_{0}\right)-d_{S}\left(x_{0}\right) \leq-h(\delta-\eta),
$$

and the proof can now proceed as before. We note that (28) does not depend on the Lipschitz constant, provided that $h$ is chosen sufficiently small. After passing to the limit as $h \downarrow 0$, therefore, 
we conclude that $(S, F)$ has an absorption rate $\delta-\eta$. By letting $\eta \downarrow 0$, we deduce that $(S, F)$ has an absorption rate $\delta$.

In the case of a general upper semicontinuous multifunction $F$, we apply a technique to approximate $F$ by Lipschitz multifunctions. See Deimling (Ref. 11). There exists a sequence of Lipschitz multifunctions $F_{j}$ that have compact convex values and satisfy

$$
F(x) \subseteq F_{j+1}(x) \subseteq F_{j}(x) \subseteq F(\{x+(1 / j) B\} \cap\{S+r B\})
$$

for $j=1,2, \ldots$ It is clear that the $\delta$ hypothesis assumed by $F$ is inherited by each $F_{j}$. The above procedure thus provides a trajectory $x_{j}(\cdot)$ of $F_{j}$ approaching $S$ at a rate $\delta$. Letting $j \rightarrow \infty$, we obtain an $\operatorname{arc} x(\cdot)$ as the uniform limit of some subsequence of $x_{j}(\cdot)$. We have that $x(\cdot)$ is also a trajectory of $\mathrm{F}$, and is being absorbed into $S$ at the rate $\delta$.

Similar considerations are operational for strong attainability.

Remark 3.2. It is interesting to consider the $\delta$ condition as hypothesized in Theorem 3.1, but in what could be considered the limiting case of $\delta=0$. It has recently been shown in (Ref. 12) that such a condition (and with the multifunction $F$ upper semicontinuous) is equivalent to the notion of weak invariance. Recall that a pair $(S, F)$ is weakly invariant if for every $x \in S$, there exists a trajectory for $F$ emanating from $x$ of arbitrarily long duration and which remains in $S$. The following corollary illustrates that this invariance criterion in (Ref. 12) can also be deduced from the attainability result Theorem 3.4. See also Haddad (Ref. 13) and Aubin (Ref. 14) for further developments regarding invariance.

Corollary 3.2. Suppose $S \subset \mathbb{R}^{n}$ is compact, and $F: \mathbb{R}^{n} \rightrightarrows \mathbb{R}^{n}$ is a multifunction with compact convex values and is upper semicontinuous on $S$. Furthermore, suppose that

$$
\inf _{v \in F(x)}\langle\xi, v\rangle \leq 0
$$

for all $\xi \in N_{S}^{P}(x)$. Then $(S, F)$ is weakly invariant.

Proof. Let $T>0$ and suppose $x \in S$. We must show there exists a trajectory $x(\cdot)$ for $F$ on $[0, T]$ satisfying $x(t) \in S$ for all $t \in[0, T]$. Let $r>0$, and extend $F$ to $S+r B$ by letting

$$
F(y)=\overline{\mathrm{co}} \bigcup\left\{v: v \in F(z), z \in \operatorname{proj}_{S}(y)\right\} .
$$

It can easily be checked that $F$ has compact convex values and is upper semicontinuous on $S+r B$. Let $\delta>0$, and define the multifunction $F_{\delta}$ on $S+r B$ by setting $F_{\delta}(y):=F(y)+\delta$ cl $B$ for $y \in S+r B$. It follows readily that $\left(S, F_{\delta}\right)$ satisfies the hypotheses of Theorem 3.4.

Now choose $M$ as in the proof of Theorem 3.1, and let

$$
0<h<(\min \{T, r\}) /(M+\delta) .
$$

Let $x_{1}(\cdot)$ be any solution of $F_{\delta}$ defined on $[0, h]$, and note that $d_{S}\left(x_{1}(h)\right) \leq h(M+\delta)<r$. By Theorem 3.4, the arc $x_{1}(\cdot)$ can be extended to be defined on an interval $\left[0, \tau_{1}\right]$ with $x\left(\tau_{1}\right) \in S$, and (by Remark 3.2) to satisfy $\tau_{1} \leq h(1+(M+\delta) / \delta$ ). We proceed in a similar manner with $x$ replaced by $x_{1}\left(\tau_{1}\right)$. In this way, a trajectory $x(\cdot)$ of $F_{\delta}$ is constructed on $[0 T]$ with the property that $x\left(\tau_{j}\right) \in S$ for a collection of $\tau_{j}$. Furthermore, the $\left\{\tau_{j}\right\}$ are dispersed throughout the interval $[0, T]$ with consecutive elements being no more than $h(1+(M+\delta) / \delta)$ apart. Taking a limit as $h \downarrow 0$ produces a trajectory of $F_{\delta}$ that stays in $S$, and taking a limit as $\delta \downarrow 0$ produces a trajectory of $F$ that stays in $S$. 


\section{Penetrative Systems}

In the previous section, we explored conditions that were used to drive solutions toward a target set. We now consider conditions for which solutions can be driven into the interior of a set. As examples illustrate (see Example 5.3 below), the penetrative set must be somewhat special besides merely having nonempty interior.

Definition 4.1. A set $S \subseteq \mathbb{R}^{n}$ is said to be epi-continuous near $x \in$ bd $S$ if there exists a unitary linear transformation $A: \mathbb{R}^{n} \longrightarrow \mathbb{R}^{n}$, a neighborhood $U \subset \mathbb{R}^{n}$ of $x$, and a continuous function $g: \mathbb{R}^{n-1} \longrightarrow \mathbb{R}^{1}$ so that $A x=\left(x^{0}, g\left(x^{0}\right)\right)$ for some $x^{0} \in \mathbb{R}^{n-1}$, and

$$
U \cap S=U \cap A^{-1}(\text { epi } g) .
$$

If $g$ can in addition be chosen Lipschitz, then $S$ is said to epi-Lipschitz near $x$.

Requiring that $A$ be unitary rather than merely invertible in the above definition is not critical, but has the advantage of preserving the geometry. In particular, one can easily verify that if $A$ is unitary and $\zeta \in N_{S}^{P}(y)$, then $A \zeta \in N_{A S}^{P}(A y)$. The main result of this section is the following.

Theorem 4.1. Suppose $S$ is epi-continuous near $x \in \mathrm{bd} S$, and $(S, F)$ satisfies the hypotheses of Theorem 3.1. Then $(S, F)$ is weakly penetrative near $x$ of a size depending on $\delta, \omega_{F}, U$, and the modulus of continuity of the function $g$, where $U$ and $g$ are as in Definition 4.1. If $S$ is in addition epi-Lipschitz, then $(S, F)$ has a rate of penetration depending on $\delta, \omega_{F}, U$, and the Lipschitz rank of $g$.

Proof. Let $x \in \operatorname{bd} S$, and let $A$ and $U$ be given as in Definition 4.1. The constants $r, c$, and $M$ are chosen as in the proof of Theorem 3.1 with the additional stipulation that $x+r \mathrm{cl} B \subset U$. Elements in the range of $A$ are represented as $A y=\left(y^{0}, y^{1}\right) \in \mathbb{R}^{n-1} \times \mathbb{R}^{1}$, where $y \in U$. Without loss of generality, we assume further that $A U=\left\{x^{0}+r^{0} B\right\} \times\left\{g\left(x^{0}\right)+r^{1} B\right\}$, where the constants $r^{0}, r^{1}>0$ satisfy $g\left(x^{0}+r^{0} B\right) \subset g\left(x^{0}\right)+r^{1} / 2 B$. For $\alpha>0$, we define

$$
S_{\alpha}:=A^{-1}\left\{\left(y^{0}, y^{1}\right) \in\left\{x^{0}+r^{0} B\right\} \times\left\{g\left(x^{0}\right)+r^{1} B\right\}: y^{1} \geq g\left(y^{0}\right)+\alpha\right\}
$$

Note that $S_{0} \cap U=S \cap U$. Also note that if $y \in U$ so that $A y=\left(y^{0}, g\left(y^{0}\right)\right)$ and $\zeta \in N_{S}^{P}(y)$, then $\zeta \in N_{S}^{P}\left(A^{-1}\left(y^{0}, g\left(y^{0}\right)+\alpha\right)\right)$ for all $\alpha<r^{1} / 2$.

Next, let $\bar{\alpha}>0$ be chosen so that $S_{\bar{\alpha}} \cap U \subset x+r B$, and so that the following holds:

$$
\min _{v \in F\left(A^{-1}\left(y^{0}, y^{1}+\alpha\right)\right)}\langle v, \zeta\rangle \leq-\delta\|\zeta\| / 2
$$

whenever $y=\left(y^{0}, y^{1}\right) \in(\operatorname{bd} S) \cap U$ with $y^{0} \in x^{0}+r^{0} B, \zeta \in N_{S}^{P}(y)$, and $\alpha \in[0, \bar{\alpha}]$. Such a choice of $\bar{\alpha}$ is possible in view of the $\delta$ assumption and the continuity of $F$. We shall want trajectories of $F$ that originate near $x$ to stay in $x+r B$, and so the requirement $\bar{\alpha}<r c / M$ is also imposed.

For $\alpha \in[0, \bar{\alpha}]$, the distance between $S$ and $S_{\alpha}$ is denoted by $\Delta(\alpha)$, which means that

$$
\Delta(\alpha):=\inf \left\{\|y-z\|: y, z \in U, \text { and } y \in S, z \in S_{\alpha}\right\}
$$

It is clear that $\Delta(\cdot)$ is continuous, strictly increasing, and $\Delta(0)=0$. Obviously one also has $\alpha \geq \Delta(\alpha)$. A lower bound of $\Delta(\alpha)$ can be obtained as follows. Let $\gamma>0$ be chosen so that 
$\omega_{g}(\gamma)<\alpha / 2$, where $\omega_{g}$ is the modulus of continuity of $g$. Then we have that

$$
\begin{aligned}
\Delta(\alpha) & \geq \inf \left\{\left\{\left\|y^{0}-z^{0}\right\|^{2}+\left|g\left(y^{0}\right)-g\left(z^{0}\right)-\alpha\right|^{2}\right\}^{1 / 2}: y^{0}, z^{0} \in x^{0}+r^{0} B\right\} \\
& \geq \begin{cases}\alpha / 2 & \text { if }\left\|y^{0}-z^{0}\right\|<\gamma \\
\gamma & \text { if }\left\|y^{0}-z^{0}\right\| \geq \gamma .\end{cases}
\end{aligned}
$$

By the nature of the sets $S_{\alpha}$, it can easily be seen for any $0 \leq \alpha<\beta \leq \bar{\alpha}$, that whenever $y \in\left(\overline{\operatorname{comp}} S_{\alpha}\right) \cap U, z \in S_{\beta}$, we have

$$
\|y-z\| \geq \Delta(\beta-\alpha)
$$

Note also that if $g$ is Lipschitz of rank $\lambda_{g} \geq 1$, then $\omega_{g}$ can be chosen so that $\omega_{g}(\rho)=\lambda_{g} \rho$, and consequently the estimate

$$
\Delta(\alpha) \geq \alpha /\left(2 \lambda_{g}\right)
$$

holds in this case.

We now proceed to construct a trajectory that will penetrate the interior of $S$. Let $N$ be a positive integer, and $\alpha_{j}:=j \bar{\alpha} / N$, for $j=1,2, \ldots, N$. To shorten the notation, we write $S_{j}$ for $S_{\alpha_{j}}$. We shall successively apply (the local version of) Theorem 3.1 to the sets $S_{j}$. The hypotheses of that theorem are satisfied in view of (30). There exists a trajectory $x_{1}(\cdot)$ for $F$ on an interval $\left[0, \tau_{1}\right]$ with $x_{1}(0)=x$ and $x_{1}\left(\tau_{1}\right) \in S_{1}$, and so that (by Corollary 3.1) $\tau_{1}:=T_{S_{1}}(x) \leq d_{S_{1}}(x) / c \leq \Delta\left(\alpha_{1}\right) / c \leq \bar{\alpha} /(c N)$. Inductively, there exists a trajectory $x_{j+1}(\cdot)$ of $F$ on an interval $\left[\tau_{j}, \tau_{j+1}\right]$ so that $x_{j+1}\left(\tau_{j}\right)=x_{j}\left(\tau_{j}\right)$ and $x_{j+1}\left(\tau_{j+1}\right) \in S_{j+1}$, and with $\tau_{j+1}:=\tau_{j}+T_{S_{j+1}}\left(x_{j}\left(\tau_{j}\right)\right) \leq(j \bar{\alpha}) /(c N)$.

Let $T_{N}:=\tau_{N} \leq \bar{\alpha} / c$, and define $x^{N}(\cdot)$ on $\left[0, T_{N}\right]$ by setting $x^{N}(t)=x_{j}(t)$ whenever $t \in\left[\tau_{j-1}, \tau_{j}\right]$ $\left(\tau_{0}:=0\right)$. Then $x^{N}(\cdot)$ is a trajectory of $F$ which stays in $S+r B$ (cf. (Ref. 15), Lemma 5.1).

Now let $N \rightarrow \infty$. We obtain another trajectory $x(\cdot)$ of $F$ defined on an interval $[0, T]$ so that $x(\cdot)$ is the uniform limit of a subsequence of the $\operatorname{arcs} x^{N}(\cdot)$, and $T$ is the limit of $T_{N}$. We show that $x(\cdot)$ penetrates the interior of $S$.

Define $\alpha:[0, T] \longrightarrow[0, \bar{\alpha}]$ by

$$
\alpha(t):=\sup \left\{\alpha \in[0, \bar{\alpha}]: x(t) \in S_{\alpha}\right\} .
$$

We claim that

$$
\alpha(t)-\alpha(s) \geq c(t-s)
$$

whenever $0 \leq s<t \leq T$. Indeed, for fixed $N$, if $\tau_{i}$ and $\tau_{j}(i<j)$ are grid points associated with the construction of $x^{N}(\cdot)$, then we have that

$$
\tau_{j}-\tau_{i} \leq(j-i) \bar{\alpha} /(c N)
$$

There exists a subsequence $\left\{N_{k}\right\}$ so that

$$
x^{N_{k}}(\cdot) \rightarrow x(\cdot) \quad \text { uniformly on } \quad\left[0, \min \left\{T, T^{N}\right\}\right],
$$

and there exist grid points $\left\{\tau_{i_{k}}\right\},\left\{\tau_{j_{k}}\right\}$ associated with $N_{k}$ such that

$$
\tau_{i_{k}} \rightarrow s \quad \text { and } \quad \tau_{j_{k}} \rightarrow t
$$


as $k \rightarrow \infty$. We have $x^{N_{k}}\left(\tau_{i_{k}}\right) \rightarrow x(s)$ and $x^{N_{k}}\left(\tau_{j_{k}}\right) \rightarrow x(t)$, and by the nature of the construction of $x^{N_{k}}(\cdot)$, we conclude that

$$
\alpha(s)=\lim _{k \rightarrow \infty} i_{k} \bar{\alpha} / N_{k} \quad \text { and } \quad \alpha(t)=\lim _{k \rightarrow \infty} j_{k} \bar{\alpha} / N_{k},
$$

where in particular the limits exist. Therefore by (34), we have

$$
\alpha(t)-\alpha(s)=\lim _{k \rightarrow \infty}\left(j_{k}-i_{k}\right) \bar{\alpha} /\left(N_{k}\right) \geq c\left(\tau_{j_{k}}-\tau_{i_{k}}\right)=c(t-s),
$$

which is the claim (33).

We now define $\rho:[0, T] \longrightarrow[0, \bar{\alpha}]$ by setting $\rho(t):=\Delta(\alpha(t))$. We have that $\rho(0)=0$, and it is immediate from the claim (33) and the definition of $\Delta$ that $\rho$ is strictly increasing. Furthermore, in the epi-Lipschitz case, from (32) and (33), it follows that

$$
\rho(t) \geq \frac{c}{2 \lambda_{g}} t
$$

Thus to finish the proof of the theorem, it is only necessary to show that

$$
x(t)+\rho(t) \operatorname{cl} B \subset S .
$$

But (36) is an immediate consequence of the definitions. Indeed, if $\|y-x(t)\| \leq \rho(t)$, then $y \in S_{0}=S$ by $(31)$.

Remark 4.1. (a) We do not go into the details here, but one can can formulate and prove similar weak penetration results akin to Theorems 3.3 and 3.4. Furthermore, strong penetration results can likewise be derived.

(b) As pointed out by L. D. Berkovitz, the convexity of the values of the multifunction $F$ can be dropped in Theorem 4.1 when $F$ is Lipschitz, since then original trajectories are dense in relaxed ones (see Ref. 10, Chapter 3]).

(c) We also mention that Rockafellar (Ref. 16) has characterized epi-Lipschitz sets in $\mathbb{R}^{n}$ as those sets $S$ that satisfy int $T_{S}^{C}(x) \neq \phi$ for $x \in \mathrm{bd} S$. Hence the following is an immediate corollary to this result and Theorem 4.1.

Corollary 4.1. Suppose $(S, F)$ satisfies the hypotheses of Theorem 3.1 and

$$
\operatorname{int} T_{S}^{C}(x) \neq \phi
$$

for each $x \in \operatorname{bd} S$. Then $(S, F)$ is weakly penetrative.

\section{$5 \quad$ Examples}

In this section, we provide some examples to justify the hypotheses in the above results.

Example 5.1. In this first example, we give a simple instance in which $F$ is upper semicontinuous and the $\delta$ condition in Theorem 3.1 is satisfied, but for which weak attainability fails. Thus continuity is required in the theorem. 
We consider $n=1, S:=[-1,1]$, and $F: \mathbb{R}^{1} \rightrightarrows \mathbb{R}^{1}$ given by:

$$
F(x)= \begin{cases}\{-1-x\} & \text { if } x<-1 \\ {[x, 0]} & \text { if }-1 \leq x \leq 0 \\ {[0, x]} & \text { if } 0 \leq x \leq 1 \\ \{1-x\} & \text { if } 1<x\end{cases}
$$

Then the trajectories of $F$ starting outside $S$ are $x(t)=1+(x-1) e^{-t}$ if $x>1$, and $x(t)=$ $-1+(x+1) e^{-t}$ if $x<-1$, which do not reach $S$ in finite time.

Example 5.2. This example shows that a uniform $\delta$ in Theorem 3.1 is necessary. Note also that while the $\delta$ hypothesis of Theorem 3.3 is not satisfied, it fails at only a single point.

Consider $n=2$ and $S:=\operatorname{cl} B \cap\{(x, y): y \geq-|x|\}$. We are only interested in the multifunction $F: \mathbb{R}^{2} \rightrightarrows \mathbb{R}^{2}$ near the origin defined by $F(x, y):=\{(0,-|y|)\}$. Any trajectory of $F$ beginning at $(0, y)$ with $y<0$ fails to reach $S$ in finite time, although for each boundary point with a proximal normal, there exists a $\delta>0$ (depending on the point) that satisfies the $\delta$ condition in Theorem 3.1

Example 5.3. This example shows that if $(S, F)$ satisfies all the hypotheses of Theorem 4.1 , but $S$ fails to be epi-continuous, then $(S, F)$ may fail to be penetrative. Note that this $S$ coincides with the closure of its interior, a hypothesis one may at first think could replace epi-continuity.

Let $n=2$,

$$
S:=\operatorname{cl} B \cap\left\{(x, y): x \leq|y|^{2}\right\} \cap\left\{(x, y): x \geq-|y|^{2}\right\},
$$

and $F$ be given by $F(x, y)=[-1,1] \times\{0\}$. Again we are only interested in the behavior of the system near the origin, in which it is clear that no trajectory beginning at a point $(x, 0)$ can enter the interior of $S$. All of the hypotheses of Theorem 4.1 are satisfied except for epi-continuity.

\section{References}

1. Petrov, N. N., Controllability of Autonomous Systems, Differential Equations Vol. 4, pp. 311-317, 1968.

2. Bardi, M., and Falcone, M., An Approximation Scheme for the Minimum Time Function, S.I.A.M. Journal of Control and Optimization Vol. 28, pp. 950-65, 1990.

3. Cannarsa, P., and Sinestrari, C., Convexity Properties of the Minimum Time Function, preprint.

4. Clarke, F. H., and Ledyaev, Yu. S., Mean Value Inequalities, Proceedings American Mathematical Society, to appear.

5. Clarke, F. H., Ledyaev, Yu. S., and Wolenski, P. R., Proximal Analysis and Minimization Principles, to appear.

6. Clarke, F. H., Methods of Dynamic and Nonsmooth Optimization, CBMS-NSF Regional Conference Series in Applied Mathematics, SIAM, Philadelphia, Pennsylvania, Vol. 57, 1989.

7. Loewen, P. D., Optimal Control via Nonsmooth Analysis, CRM Proceedings and Lecture Notes, American Mathematical Society, Providence, Rhode Island, 1993. 
8. Clarke, F. H., and Ledyaev, Yu. S., Mean Value Inequalities in Hilbert Space, Transactions of the American Mathematical Society, Vol. 344, pp. 307-324, 1994.

9. Clarke, F. H., Stern, R. J., and Wolenski, P. R., Proximal Smoothness and the Lower$C^{2}$ Property, Journal of Convex Analysis, to appear.

10. Clarke, F. H., Optimization and Nonsmooth Analysis, Wiley-Interscience, New York, New York, 1983; Second Edition: SIAM, Philadelphia, Pennsylvania, Classics in Applied Mathematics, Vol. 5, 1990.

11. Deimling, K., Multivalued Differential Equations, De Gruyter, Berlin, Germany, 1992.

12. Clarke, F. H., Ledyaev, Yu. S., Stern, R. J., and Wolenski, P. R., Qualitative Properties of Differential Inclusions: a Survey, Journal of Dynamical and Control Systems, to appear.

13. Haddad, G., Monotone Trajectories of Differential Inclusions and Functional Differential Inclusions with Memory, Israel Journal of Mathematics, Vol. 39, pp. 83-100, 1981.

14. Aubin, J.-P., Viability Theory, Birkhauser, Boston, Massachusetts, 1991.

15. Wolenski, P. R., A Uniqueness Theorem for Differential Inclusions, Journal of Differential Equations, Vol. 84, pp. 165-182, 1990.

16. Rockafellar, R. T., Clarke's Tangent Cones and the Boundaries of Closed Sets in $\mathbb{R}^{n}$. Nonlinear Analysis, Vol. 3, pp. 145-154, 1979. 